\title{
Automatic Detection, Extraction and Analysis of Unrestrained Gait Using a Wearable Sensor System
}

\author{
Amin Ahmadi $^{1}$, Chris Richter ${ }^{2}$, Noel E. O'Connor ${ }^{1}$ and Kieran Moran ${ }^{3}$
}

\begin{abstract}
Within this paper we demonstrate the effectiveness of a novel body-worn gait monitoring and analysis framework to both accurately and automatically assess gait during 'freeliving' conditions. Key features of the system include the ability to automatically identify individual steps within specific gait conditions, and the implementation of continuous waveform analysis within an automated system for the generation of temporally normalized data and their statistical comparison across subjects.
\end{abstract}

\section{INTRODUCTION}

Gait analysis is used in the clinical management and scientific research of pathological gait related to neuromuscularskeletal damage and cognitive decline [1], [2], to facilitate early screening, enhance intervention decision-making and track long-term deterioration. Gait analysis can incorporate joint kinematics (angle and angular velocity) and spatiotemporal measures (step length, step duration, foot trajectory) [3].

Gait analyses are traditionally undertaken in controlled laboratory/clinical environments using expensive walkway mats (e.g. GaitRite) or camera-based systems (e.g. Vicon). However, analyses are not widespread because of system costs and availability. More importantly, the ecological validity of the results is questionable because of the small number of foot strikes $(<5)$, the very controlled environment where participants are highly focused and only straight line walking is assessed [1]. Given the implicit aim of gait analysis is to capture a person's natural gait, it would be far more appropriate to assess walking over a much longer time/distance and, most critically, under 'free-living' conditions.

The use of small, light, wireless inertial sensors in a body-worn monitor (defined as the sensors and associated algorithms) offers a relatively cheap and accurate alternative [7]. A number of systems have been proposed that examine issues of accuracy in spatial-temporal measures [13] and joint kinematics [12] and are beginning to be more widely employed within research and lifestyle-based intervention studies. However, a major challenge is that 'freeliving walking involves periods of acceleration (e.g. initiating walking; after opening a door), deceleration (e.g. walking

\footnotetext{
${ }^{1}$ Amin Ahmadi and Noel E.O'Connor are with the Insight Center for Data Analytics, Dublin City University, Dublin, Ireland [amin.ahmadi, Noel.oconno] ddcu.ie

${ }^{2}$ Chris Richter is with the Department of Sport Medicine, Sports Surgery Clinic, Dublin, Ireland ChrisRichter@sportssurgeryclinic.com

${ }^{3}$ Kieran Moran is with the Applied Sports Performance Research School of Health and Human Performance, Dublin City University, Dublin, Ireland kieran.moran@dcu. ie
}

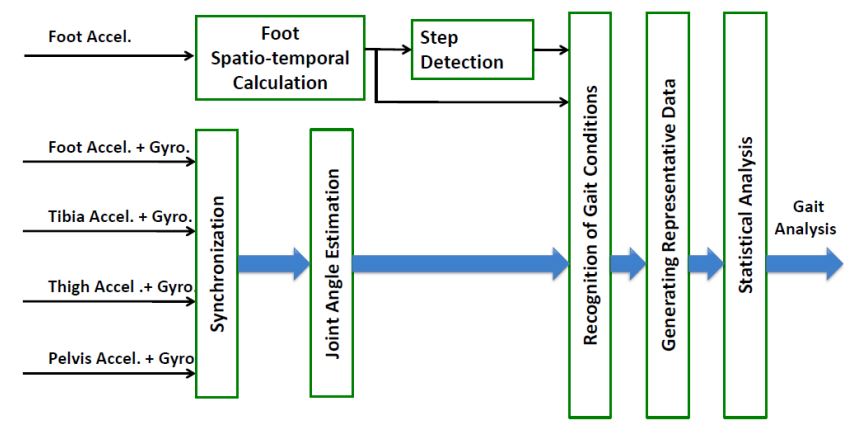

Fig. 1: Main components of the proposed framework.

within a crowd; coming to a closed door), turning corners, and ascending/descending curbs and stairs; with all of these walking conditions affecting gait spatio-temporal and joint kinematics. Given that intra-patient and inter-patient comparisons are central to gait analysis, it is essential to only compare across the same walking conditions. Therefore it is necessary for body-worn gait monitoring systems to automatically identify each walking condition and extract each gait cycle.

Although kinematic measures are continuous in nature, they are currently generally analyzed by extracting key discrete events (e.g. maximum knee flexion); this can significantly undermine the accuracy of the findings [4]. Clearly it would be more informative for a body-worn gait monitoring system to automatically create and analyze representative data across the whole gait step cycle.

Within this paper we propose a novel body-worn gait monitoring and analysis framework to both accurately and automatically assess gait during 'free-living' conditions.

\section{FRAMEWORK}

The main components of our framework (Fig. 1) are: synchronization, calculation of joint angle and spatio-temporal measures, step detection, recognition of gait condition, generating representative data and statistical analysis. Each component is presented and discussed below.

\section{Methodology}

\section{A. Data Collection}

To evaluate the proposed framework, five healthy participants $(37 \pm 7$ years; $1.76 \pm 5 \mathrm{~m} ; 77 \pm 7 \mathrm{~kg}$ ) were compared to one patient with gait impairment (29 years; $1.88 \mathrm{~m} ; 82 \mathrm{~kg}$ ) left sided hemiplegia (occurring prenatal). The patient was highly functional, representing Ireland in multiple running distances. The non-injured right side of the patient was 
analyzed in order to provide a greater challenge to the system to statistically identify gait kinematic differences.

Movement kinematics were assessed using four wearable inertial sensors (x-IMU, $\mathrm{x}$-io Technologies, UK) strapped to the participants' mid-foot, shank, thigh, and pelvis with elastic Velcro straps (Fig. 2). The x-axes were aligned with the longitudinal axes of the body segments.

Participants performed a walk along a predefined path to mimic various free-living conditions (Fig. 3), including: gait initiation/acceleration and deceleration, changes in direction, straight line walking, opening doors, ascending and descending stairs, and semi-stationary standing (as if at a shop counter). The path was walked in both directions (returning to the start). Walking speed was self-selected. Annotated video along with the calculated location of the foot along the gait path, was used as the ground truth for evaluating the automatic segmentation and recognition of gait conditions. The experimental procedures involving human subjects described in this paper were approved by the Institutional Review Board.

\section{B. Synchronization}

An internal SD card was used to store data from each sensor $(256 \mathrm{~Hz})$. A physical event (5 repeated stiff jumps to generate high impact accelerations simultaneously in all sensors at the start and end of the walking trial) was used to help temporally synchronize all sensors. This was achieved by automatically identifying the first acceleration peak associated with the first stiff landing from all sensors prior to walking. The effectiveness of this was evaluated by comparing the last acceleration peak from the fifth stiff landing from all sensors immediately after the walking trial. The system was $100 \%$ successful in synchronizing.

\section{Calculation of Joint Angle and Spatio-temporal Measures}

We use a customized gradient descent optimization algorithm [5], [7] on three-dimensional (3D) accelerometer and gyroscope data to estimate the sensor orientation with respect to the Earth frame. This algorithm has been shown to provide effective performance at low computational expense and is as accurate as the Kalman based algorithm [5]. Each sensor unit

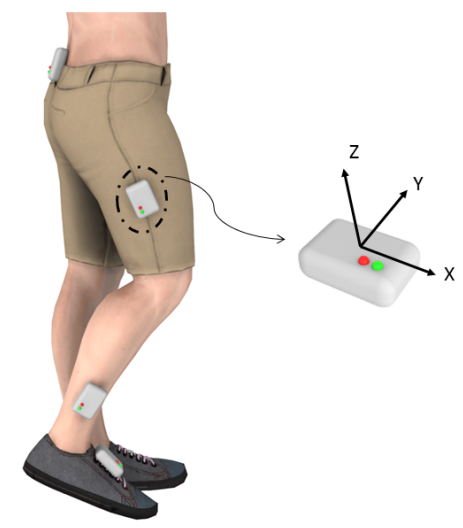

Fig. 2: Placement of four inertial sensor units.

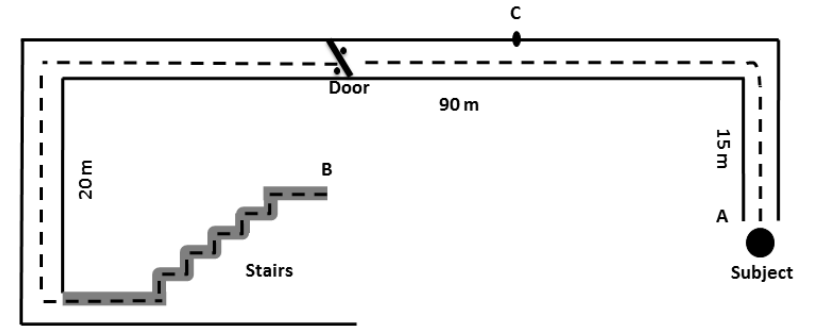

Fig. 3: Walking path (A to B and back to A). Walking from B to $\mathrm{A}$, the door was open; participants were in semi-stationary standing at point $\mathrm{C}$ (20 seconds).

frame was aligned with the body frame [6] and a technique described in [7], [8] was then applied to the foot, shank, thigh and pelvis segments to measure ankle, knee and hip flexionextension joint angles. In theory, double integration of the acceleration measurements should yield position. However, due to accelerometer drift error, position estimates are only valid for a short period of time and require drift correction [9], [10].

During early stance, the total acceleration of the foot sensor is very small (almost zero) indicating when the initial velocity can be set to zero; and consequently the $3 \mathrm{D}$ position of the foot during the swing phase can be calculated [11], (Fig. 4).

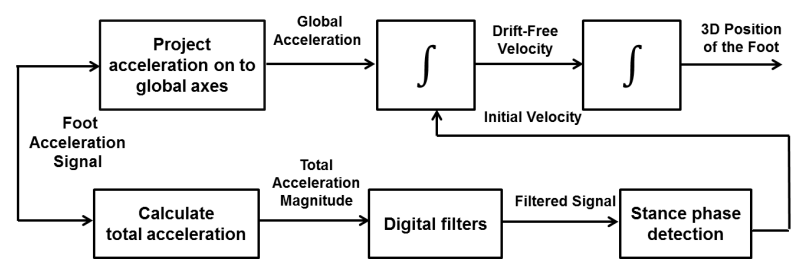

Fig. 4: Overview of the foot position estimation process.

\section{Data Analysis}

Although a large number of kinematic measures and walking conditions were included, we limit our analysis of continuous variables here to sagittal plane flexion-extension and foot z-position, during periods of straight-line walking that did not involve stair climbing or acceleration.

1) Step Detection: Step cycles were defined as heel-strike to heel-strike. Heel strike detection employed two phases. Firstly, we calculated the moving standard deviation $(M S D)$ of the vertical velocity of the foot sensor (in the global frame) over a moving window of 100 frames $(0.39$ seconds). The $M S D$ was used to identify where the foot was in a stationary foot position (i.e. flat foot of the stance phase). At every frame $(t)$, the foot was defined as stationary and stored into a StepIDX if (1) was satisfied:

$$
M S D_{t}>[\min (M S D)+\operatorname{range}(M S D) \times 0.5]
$$

Secondly, we selected the root mean square value of the vertical acceleration at the start of every defined stationary foot position as the threshold to detect heel strike (i.e. the 


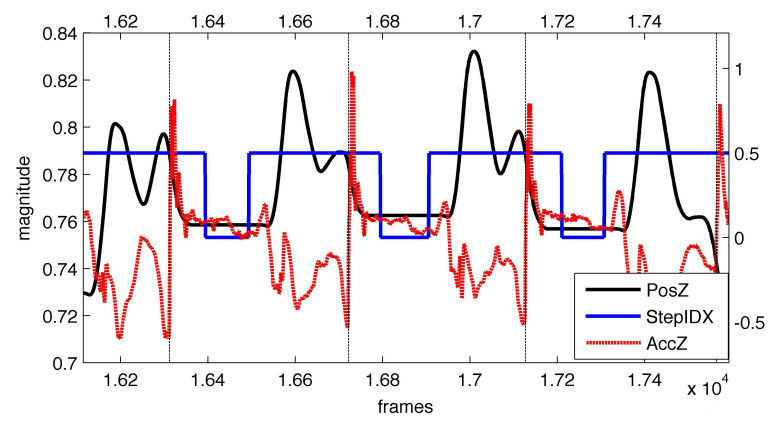

Fig. 5: Step detection using the foot sensor.

last value above this threshold) (Fig. 5). The position (x, y and $\mathrm{z}$ ) and coordination angle of the foot sensor were zeroed using the value at $20 \%$ of the movement cycle.

2) Recognition of Gait Conditions: Using the z-position of the foot sensor, the correlation coefficient $(r)$ was calculated between the step mean and the individual $\mathrm{z}$ values during each step cycle. Correlation coefficients of less than 0.8 identified phases of standing, acceleration (opening/closing doors) and stair climbing. Periods of curved walking (turning corners or swerving to avoid other people) were identified by an absolute coordinate angle of the foot greater than 0.1 radian in the ground (horizontal) plane. In addition, based on pilot testing, three steps before and after curved walking, stair climbing, opening doors and being stationary were removed as further periods of acceleration/deceleration. The remaining cycles were defined as straight line walking at relatively constant speed, and analyzed.

3) Generating Representative Data Following Temporal Normalization: Walking speed affects joint and foot kinematics. To remove temporal variations all kinematic curves were landmark registered to key events [15]. Firstly, the following landmarks were identified: maximum hip, knee and ankle angles, and tested for and removed outliers [14]. Time warp functions were then created by fitting each cycle's landmark sequence to the mean landmark sequence using a polynomial fit (4th order) for every cycle. Finally, the hip, knee and ankle angles as well as z-position and the normalized percentage $(1: 100)$ of every cycle were registered using its corresponding time warp function, utilizing Cubic Spline data interpolation to generate representative data for both the normal gait and the hemiplegic (impaired) gait.

4) Statistical Analysis: To examine statistical differences in the continuous data (joint angles and foot z-position) between healthy participants and the patient, we applied a continuous waveform analysis recently developed by our group [Analysis of Characterizing Phases (ACP)] [4]. The ACP used $99 \%$ of the variance in the data to generate subject scores to examine the full area over which participants differed [16]. Statistical differences in the ACP subject scores, as well as the discrete spatio-temporal measures (step length; step duration), were examined using independent t-tests or Mann-Whitney U test when non-parametric. $(p<.05$ was adopted). Cohen's D was calculated to examine the effect size.
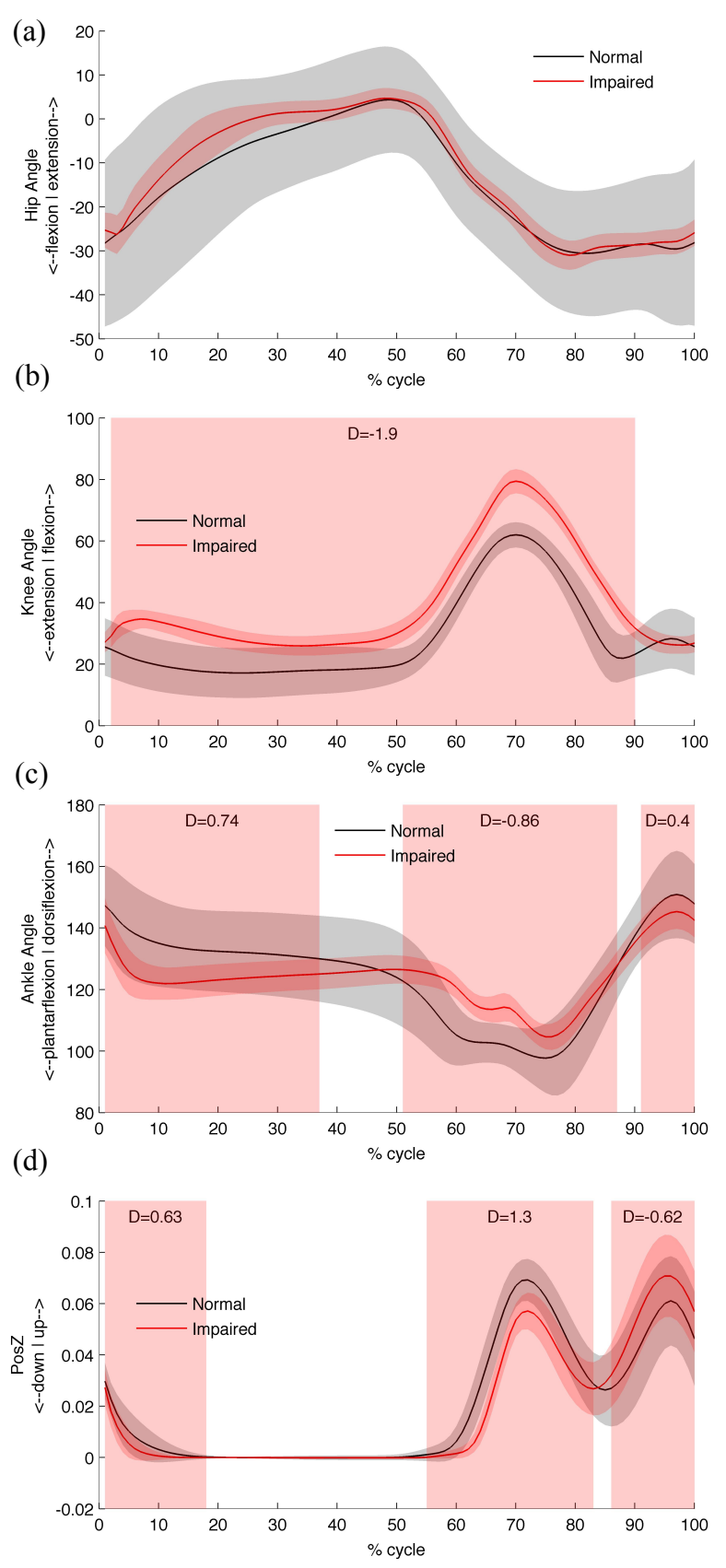

Fig. 6: Registered means and confidence intervals for (a) hip, (b) knee, (c) ankle, angle, and (d) foot vertical height, for normal versus impaired walking. Phases of significant differences are indicated by the vertical red bands. D is the Cohen's effect size.

\section{Results AND Discussion}

In comparing the identified walking actions to the ground truths from both the video and the calculated location of the foot along the preplanned gait path, the system was able to automatically exclude phases of curved walking, climbing stairs, acceleration/deceleration, and semi-stationary standing). Within 470 detected steps of straight line walking, 3 cycles were incorrectly included (unusual long steps during 
acceleration) and subsequently manually removed prior to statistical analysis. [Their inclusion however would not have altered the general outcomes of the analysis]. Identifying and grouping different walking conditions is extremely important in the generation and statistical analysis of representative data given that they will utilize different joint and foot kinematics. Without this function, it is not possible to effectively compare across participants (as in this study) or to longitudinally track individuals for the early identification/screening of neuro-musculoskeletal injury or cognitive decline [1], [2]. Currently, no systems appear to facilitate this.

Representative data for joint flexion-extension and for the vertical position of the foot are depicted (Fig. 6). All graphs exhibit the classic shapes for gait, testifying to the accuracy of the implemented joint angle and spatio-temporal algorithms. Given that the 'unaffected' side of the hemiplegic gait was analyzed, which has smaller differences to normal gait [17] than the affected side, the clear statistically significant differences identified lend support to the processes used here in generating and analyzing the representative data. In particular the use of continuous waveform analysis (rather than discrete points), which has been previously shown to be superior [4], [16] is shown to be extremely important.

The hemiplegic gait demonstrated more pronounced $(0-$ $36 \%)$ and higher rates $(0-10 \%)$ of passive plantar flexion during stance, which may be associated with a higher and faster transition off of the affected side, which is characteristically very rigid and has a shorter stance time [17], [1]. During early swing $(51-88 \%)$ the patient used more ankle dorsiflexion, which may reflect a strong desire to avoid hemiplegic associated tripping [1]. The greater knee flexion throughout almost the entire gait cycle $(0-90 \%)$ may be due to hip hitching on the affected side forcing the knee into more flexion due to inter-segmental reactions. Flexionextension of the hip did not differ. Differences in the vertical position of the foot were reflective more of the differences in ankle dorsiflexion than knee flexion. For hemiplegic gait the step length was longer but the step duration was shorter (Fig. 7 ). The former may be due to rocking over the more rigid and straighter affected (left) side or simply because he was taller than the normal participants. The shorter step duration requires further investigation.

While the proposed framework was evaluated in a 'freeliving' condition, not all free-living conditions were included (e.g. outdoor and uneven ground, complex crowds). Further work is needed in this regard.

\section{ACKNOWLEDGMENT}

The research leading to these results has received funding from the European Community's Seventh Framework Programmes (FP7/2013-2016) under grant agreement no. ICT2011-8.2.601170 (REPLAY project).

\section{REFERENCES}

[1] Kinsella, S., Moran, K. (2008). Gait pattern categorization of stroke participants with equinus deformity of the foot. Gait and posture, 27(1), 144-151. (a)
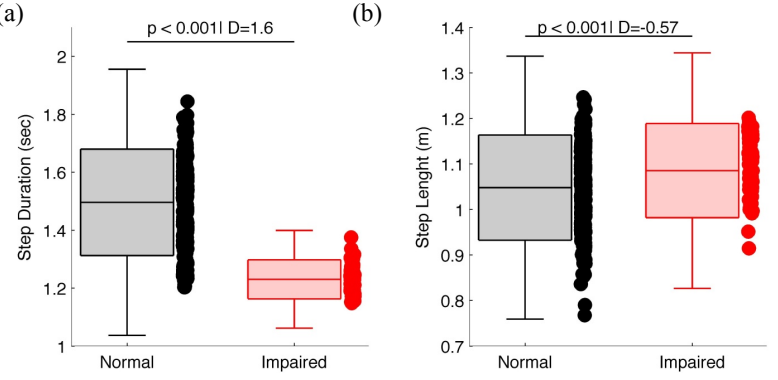

Fig. 7: Boxplot differences for (a) step duration and (b) step length. $\mathrm{p}$ is the likelihood of significant difference and $\mathrm{D}$ is the Cohen's effect size.

[2] Verghese, J., Wang, C., Lipton, R. B., Holtzer, R., Xue, X. (2007). Quantitative gait dysfunction and risk of cognitive decline and dementia. Journal of Neurology, Neurosurgery and Psychiatry, 78(9), 929-935.

[3] Cimolin, V., Galli, M. (2014). Summary measures for clinical gait analysis: A literature review. Gait and posture, 39(4), 1005-1010.

[4] Richter, C., Marshall, B., Moran, K. (2014). Comparison of discrete-point vs. dimensionality-reduction techniques for describing performance-related aspects of maximal vertical jumping. Journal of biomechanics, 47(12), 3012-3017.

[5] Madgwick, S. O., Harrison, A. J., and Vaidyanathan, R. (2011, June). Estimation of IMU and MARG orientation using a gradient descent algorithm. In Rehabilitation Robotics (ICORR), 2011 IEEE International Conference on (pp. 1-7).

[6] Favre, J., Aissaoui, R., Jolles, B. M., De Guise, J. A., Aminian, K. (2009). Functional calibration procedure for 3D knee joint angle description using inertial sensors. Journal of biomechanics, 42(14), 2330-2335.

[7] Ahmadi, A., Mitchell, E., Richter, C., Destelle, F., Gowing, M., OConnor, N. E., Moran, K. (2015). Towards Automatic Activity Classification and Movement Assessment During a Sports Training Session. IEEE Internet of Things Journal, 2(1), 23-32

[8] Roetenberg, D., Luinge, H., Slycke, P. (2009). Xsens MVN: full 6 DOF human motion tracking using miniature inertial sensors. Xsens Motion Technologies BV, Tech. Rep.

[9] Mariani, B., Rochat, S., Bula, C. J., Aminian, K. (2012). Heel and toe clearance estimation for gait analysis using wireless inertial sensors. Biomedical Engineering, IEEE Transactions on, 59(11), 3162-3168.

[10] Ahmadi, A., Destelle, F., Monaghan, D., OConnor, N. E., Richter, C., Moran, K. A Framework for Comprehensive Analysis of a Swing in Sports Using Low-Cost Inertial Sensors. In Sensors, 2014. 13th IEEE Conference on (pp. 2211-2214). IEEE.

[11] Yun, X., Bachmann, E. R., Moore, H., Calusdian, J. (2007, April). Self-contained position tracking of human movement using small inertial/magnetic sensor modules. In Robotics and Automation, 2007 IEEE International Conference on (pp. 2526-2533)

[12] Dadashi, F., Mariani, B., Rochat, S., Bla, C. J., Santos-Eggimann, B., Aminian, K. (2013). Gait and foot clearance parameters obtained using shoe-worn inertial sensors in a large-population sample of older adults. Sensors, 14(1), 443-457.

[13] Sabatini, A. M., Martelloni, C., Scapellato, S., Cavallo, F. (2005). Assessment of walking features from foot inertial sensing. Biomedical Engineering, IEEE Transactions on, 52(3), 486-494.

[14] Barnett, V., Lewis, T. (1994). Outliers in statistical data (Vol. 3). New York: Wiley.

[15] Ramsay, J. O. (2006). Functional data analysis. John Wiley and Sons.

[16] Richter, C., McGuinness, K., OConnor, N. E., Moran, K. (2014). The Variance Needed to Accurately Describe Jump Height From Vertical Ground Reaction Force Data. Journal of applied biomechanics, 30(6), 732-736.

[17] Olney, S. J., Griffin, M. P., McBride, I. D. (1994). Temporal, kinematic, and kinetic variables related to gait speed in subjects with hemiplegia: a regression approach. Physical Therapy, 74(9), 872-885. 